\title{
Arthroderma gypseum Y SU ANAMORFO EN EL JARDIN CENTRAL DE LA UNIVERSIDAD NACIONAL DE TUCUMAN, ARGENTINA.
}

\author{
(Artroderma gypseum and its anamorph from the central garden of the \\ Universidad Nacional deTucuman, Argentina)
}

\author{
Aida van Gelderen de Komaid \& Isabel Borges de Kestelman \\ Cátedra de Micología, Instituto de Microbiología «Luis C. Verna», \\ Facultad de Bioquímica, Química y Farmacia, Universidad Nacional de Tucumán, \\ Ayacucho 491, (4000) San Miguel de Tucumán, \\ República Argentina. micologia@fbqf.unt.edu.ar
}

Palabras Claves: Hongos geofílicos, Arthroderma gypseum, Tucumán, Argentina. Key words: Geophilic fungi, Arthroderma gypseum, Tucumán, Argentina.

RESUMEN

Con el objeto de contribuir al conocimiento de la micobiota en áreas públicas, en especial en la búsqueda de propágulos infectivos para el hombre y los animales de dermatofitos geofílicos desde sus fuentes saprofíticas, se estudiaron en el lapso de 2 años, 8 muestras superficiales de suelo (una cada 3 meses) desde los jardines centrales de la Universidad Nacional de Tucumán mediante la Técnica de Vanbreuseghem, usando pelos de niños como anzuelo queratínico. Las 8 muestras sembradas en 24 placas de Petri, presentaron todas al cabo de 25-30 días la presencia de Arthroderma gypseum y su anamorfo Microsporum gypseum como único dermatofito presente. Los cultivos en Sabouraud glucosado y en Sabouraud al 10\% (medio deTakashio), permitieron identificar las cepas típicas del anamorfo. La presencia y prevalencia en el tiempo de este agente en las muestras colectadas en dependencias universitarias rodeadas de aulas, laboratorios y oficinas, posee un impacto en salud pública y ambiental por su conocido oportunismo en pacientes inmunocompetentes e inmunocomprometidos.

\section{INTRODUCCION}

Los dermatofitos son hongos especializados queratinofílicos y queratinolíticos que atacan tejidos

Recibido el 22 de Octubre 2007

Aceptado el 4 de Diciembre 2007
ABSTRACT

With the purpose of knowing the presence of mycobiota in public areas, mainly in the search of propagules of geophilic dermatophytes which are infective for man and animals from their saprophytic sources, 8 superficial samples of soil (every 3 month), collected from the central gardens of the Universidad Nacional de Tucumán were studied during a 2-year period, by using the Vanbreuseghem Technique which employs children hair as a keratinic bait. The overall 8 samples sowed on 24 Petri plates revealed after 25-30 days the presence of Arthroderma gypseum and its anamorph Microsporum gypseum as the only dermatophyte present. Cultures in glucose Sabouraud and in 10\% Sabouraud (Takashio medium), allowed the identification of the typical strains of the anamorph. Presence and prevalence in time of this agent in samples collected from university buildings surrounded by classrooms, laboratories and offices means an impact on public and environmental health due to its well known opportunism in immunocompetent and immunocompromised patients.

queratinizados (piel, pelos y uñas) del hombre y otros vertebrados, causando infecciones superficiales denominadas «tineas» o comúnmente tiñas. Pertenecen a tres géneros anamórficos: Microsporum, Trichophyton y Epidermophyton, dentro del Phylum Ascomycota (Eurotiomycetes, Onygenales) (1). Algunas especies tienen un estado teleomórfico en el género Arthro- 
derma, en la Familia Arthodermataceae (2, 14). Este estado se caracteriza por la formación de esporas sexuadas contenidas en cuerpos de fructificación (ascocarpos) laxos llamados gymnotecios $(3,6)$. De acuerdo a su habitat natural, los dermatofitos se dividen en 3 grupos: geofílicos, zoofílicos y antropofílicos.

Las lesiones en la piel y sus anexos en humanos, son causadas por especies antropofílicas, por algunas especies zoofílicas y menos frecuentemente geofílicas. Pueden variar desde lesiones descamativas benignas a muy severas con marcada reacción inflamatoria y/o supuración dependiendo de la reacción del hospedador a los productos metabólicos del hongo, del sitio de la lesión, de la virulencia de la cepa y de factores locales o asociados a deficiencias del sistema inmune (3, 4, 5, 6).

Los residuos de queratina del suelo, a expensas de los cuales pueden desarrollarse los dermatofitos, permiten que este se comporte como reservorio permanente u ocasional de estos hongos y como fuente potencial de infección para humanos y animales $(4,6)$.

Arthroderma gypseum (Nannizzi) Weitzman et al. y su anamorfo Microsporum gypseum (Bodin) Guiart \& Grigorakis, es común en suelos ricos en humus, y puede comportarse ocasionalmente como zoofílico o antropofílico cuando se asocia a la queratina humana y animal, produciendo lesiones superficiales en la piel y sus anexos.

M.gypseum, es la especie geofílica más común en dermatofitosis humanas con variada frecuencia. Puede causar tinea capitis inflamatorias o no inflamatorias en niños y raramente adultos, tinea corporis en adultos y niños y solo excepcionalmente ataca las uñas $(4,5,6)$. Su virulencia puede incrementarse al asociarse a hospederos con compromiso inmune, dando lesiones a veces atípicas $\mathrm{y}$, en casos raros, es agente de micosis profundas subcutáneas (3, 6, 7, 8, 9, 10, 11).

M. gypseum, es un hongo heterotálico cuya sexualidad se expresa por la fusión de talos compatibles $(+/-)$, que bajo determinadas condiciones de cultivo dan lugar al holomorfo con producción de conidios y ascosporas en ascocarpos laxos (gymnotecios) $(4,6,12)$.

En este trabajo se investigó la presencia en el suelo de dermatofitos potencialmente patógenos para el hombre en los jardines de nuestra Casa Central con el objeto de contribuir al conocimiento de la micobiota queratinofílica y queratinolítica de espacios públicos.

\section{MATERIALES Y METODOS}

Muestras de suelo. Se estudiaron 8 muestras de suelo de aproximadamente 500 g c/u (4 muestras/año, una cada 3 meses) entre setiembre del 2003 y 2005, tomadas al azar en varios puntos de la capa superficial de los jardines de la UNT en un área aproximada de $50 \mathrm{~m}^{2}$ cada una, juntándoselas en un pool en bolsas plásticas. Las siembras fueron realizadas en 3 placas de Petri por muestra, siguiendo la Técnica de Vanbreuseghem, usando trozos $(1-2 \mathrm{~cm})$ de pelos de niños como anzuelos de queratina (13). Las placas fueron mantenidas humedecidas con solución de antibacterianos a razón de $50 \mathrm{U} / \mathrm{mL}$ de penicilina y $40 \mu \mathrm{g} / \mathrm{mL}$ de estreptomicina, incubándose a $28^{\circ} \mathrm{C}$, por 2 meses. Durante ese tiempo, para mantener la humedad de los suelos, se agregó agua destilada estéril toda vez que fue necesario.

Medios de cultivos. La presencia fúngica en las placas de Petri fue detectada bajo la lupa y por microscopía. Todas las cepas fueron aisladas estudiándose las características diferenciales del desarrollo de las colonias de sus anamorfos en placas con Sabouraud glucosado y el medio de Takashio (Sabouraud diluido 1/10 con sales, conteniendo $2 \mathrm{~g}$ de glucosa, $1 \mathrm{~g}$ de neopeptona, $1 \mathrm{~g}$ de MgSO4 7H2O2, $1 \mathrm{~g}$ de KH2PO4, 20 g de agar y 1000 mL de agua destilada), incubándose a $28^{\circ} \mathrm{C}$ durante $10-15$ días $(21,22)$.

Identificación de los aislamientos: La identificación de las cepas fue realizada mediante características micro y macromorfológicas directamente sobre el anzuelo queratínico con preparaciones en Lactofenol con azul de algodón y por cultivos, según la técnica de Stockdale (12), pero en especial la de Takashio $(20,21)$ además de la producción de órganos perforadores de pelos «in vitro» $(3,4,5)$. El estado teleomórfico dentro del complejo M.gypseum (Arthroderma gypseum, A. uncinatum, A. fulvum y A.corniculatum), se analizó en el substrato queratínico, teniendo en cuenta las características de los cuerpos de fructificación desarrollados (gymnotecios): tamaño, color, consistencia, morfología de las hifas peridiales, características de los ascos y características de las ascosporas.

\section{RESULTADOS}

En las 8 muestras procesadas (24 placas) durante los 2 años de estudio, se obtuvo abundante desarrollo de $\boldsymbol{M}$. gypseum, siendo el único dermatofito aislado en el $100 \%$ de las 24 placas. El crecimiento del hongo sobre los anzuelos de queratina, permitió observar la morfología típica primeramente del anamorfo entre los 20 y 25 días de incubación, caracterizada por la formación de un micelio aéreo hialino, tabicado, con presencia de hifas en raqueta, abundantes hifas conidiógenas ramificadas y de compleja estructura, lisas, terminando en racimos de macroconidias de forma elipsoidal, de paredes delgadas y de extremo libre aguzado de base trunca, de 9 - 15 x 25 - $60 \mu \mathrm{m}$, presentando hasta 6 tabiques transversales (Fig. 1,3). Microconidios escasos en algunas cepas, sésiles o sobre un corto 

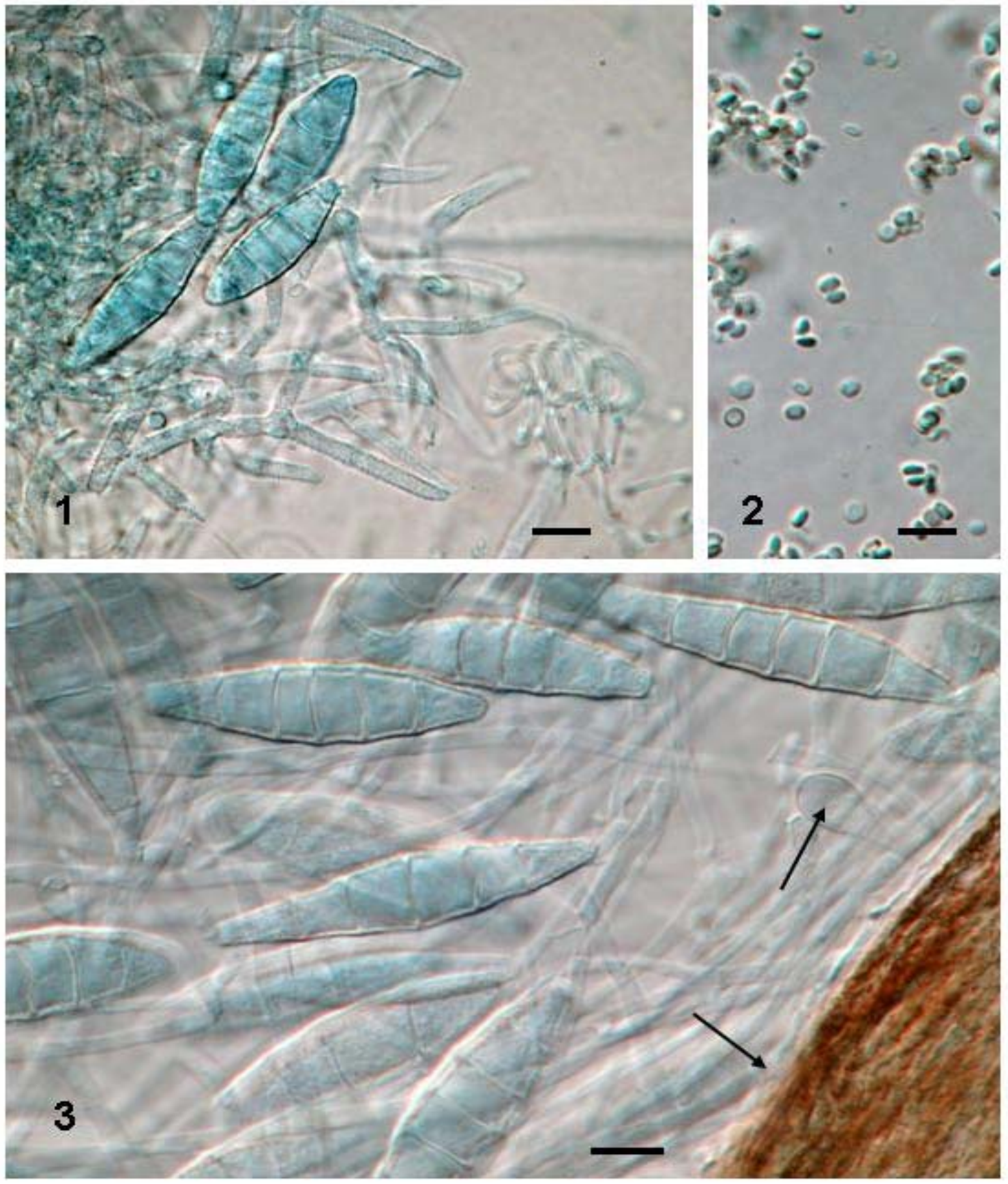

Figura 1 Arthroderma gypseum. 1,1. Parte del peridio mostrando hifas peridiales hialinas, con ramas derechas, verticiladas, verrucosas y lisas, con apéndices espiralados y la presencia de 4 conidios del anamorfo (M.gypseum). 1,2. Ascosporas lisas, redondas a elípticas en visión lateral. 1,3. Macroconidios del anamorfo sobre el pelo (flecha) y desarrollo inicial de un macroconidio (flecha). Barra $10 \mu \mathrm{m}$.

pedúnculo, generalmente de forma clavada, de paredes delgadas, lisos, 3,5-7 x 2-3 $\mu \mathrm{m}$. Todas las cepas presentaron órganos perforadores «in vitro».

A partir de 25-30 días de incubación, en los anzuelos queratínicos, se hizo evidente la formación del teleomórfo (Arthroderma gypseum), en la mayoría de las placas, caracterizado por la formación de gymnotecios blancos a cremosos, de 350-700 ìm de diámetro (Fig.1,1). Los ascocarpos se presentaron rodeados por una pared constituida por hifas peridiales hialinas, de pared gruesa, 
ligeramente verrucosas, curvadas, ramificadas de manera verticilada, compuesta por células sin septos que presentan constricciones centrales entre tabiques en forma simétrica con presencia de hifas espiraladas en el peridio (Fig. 1,1). La ruptura, por aplastamiento de los gimnotecios maduros, liberó numerosos ascos globosos, evanescentes, de paredes delgadas, de 5 a 7 ìm de diámetro con ocho ascosporas lenticulares lisas, de 1,5-2 x 2,5-3,5 ìm (Fig. $1,2)$.

Los cultivos en agar Sabouraud presentaron mayor cantidad de micelio aéreo y mostraron algunas diferencias apreciables solo en el tamaño de las colonias y su textura, mientras las sembradas en el medio de Takashio, por ser un medio pobre, mostró colonias reducidas con escaso micelio aéreo, con concentraciones de fructificaciones en el centro de las colonias que se extendieron en forma estrellada radialmente; Macroconidios simétricos, granulosos en toda su superficie externa y ampliamente elipsóides, con un apice aguzado desarrollándose en grupos terminales desde conidióforos muy ramificados (29-58 x 8-12 $\mu \mathrm{m})$.

\section{DISCUSION}

El suelo del jardín central de la UNT contiene un alto contenido del hongo potencialmente patógeno $\boldsymbol{M}$. gypseum, concordando con lo determinado en los suelos de escuelas de la provincia de Tucumán donde se comprobó la presencia de esta especie en el 86,6 \% de las muestras y un $94,4 \%$ de las muestras de los suelos de plazas y parques de la ciudad capital de la provincia (San Miguel de Tucumán) $(15,16)$. Su abundante presencia en el suelo, tiene importancia clínica, porque esta especie fue el agente del $37 \%$ de las tinea capitis de niños de Tucumán durante el período 1965-1975 (17) y del 3,3 \% de las diagnosticadas entre 1975 y 2005 (datos no publicados). La marcada disminución de su incidencia en tinea capitis, durante ambos períodos, probablemente se deba al hecho que a partir de la década del 80 los pacientes de la periferia de la ciudad y los rurales son atendidos en el Primer Nivel de Atención (Centros de Atención Primaria de la Salud), por lo tanto, en el segundo período se incluyen solamente los casos procedentes de la ciudad Capital, que poseen mejores condiciones socioeconómicas. La mayoría de las tinea capitis por $\boldsymbol{M}$. gypseum diagnosticadas fueron no inflamatorias (86,7 \%) (17), a pesar de que los dermatofitos geofílicos tienden a producir lesiones más inflamatorias que los antropofílicos. También M. gypseum tiene importancia en la región como agente de tinea corporis con incidencia del $6,9 \%(17,18)$.

La formación del teleomorfo de $M$. gypseum en el suelo, involucra la presencia de 2 talos compatibles y la existencia de ambos en el jardín de la UNT es un hecho poco frecuente en suelos de Tucumán, donde Arthroderma gypseum fue observado sólo en el $20 \%$ de las muestras (15). Esta situación puede asociarse a la mayor presencia de humus en los suelos, el $\mathrm{pH}$ favorable y al aporte queratínico de la población de estudiantes (19) y a una ditribución equitativa de ambas polaridades.

La determinación de la existencia en suelo de cepas pertenecientes a talos compatibles, de diferente sexualidad, posee también importancia epidemiológica ya que ambos talos son patógenos para el hombre (20).

El crecimiento saprofítico del hongo en el suelo forma diversos propágulos infectivos, donde los conidios (macro y microconidios) son los de mayor importancia. Su presencia en dependencias universitarias ubicadas en el radio céntrico de la ciudad y rodeada de aulas, laboratorios y oficinas, posee un impacto ambiental que plantea al mismo tiempo la necesidad de considerar su rol como potencial patógeno en pacientes inmunocompetentes e inmunocomprometidos.

\section{AGRADECIMIENTOS}

El trabajo fue desarrollado en el marco del Proyecto «Los hongos en Tucumán: sus implicancias en salud pública» subsidiado por el Consejo de Investigaciones de la Universidad Nacional de Tucumán (C.I.U.N.T. Proyecto 26 D/338) y el Consejo Nacional de Investigaciones Científicas y Técnicas (C.O.N.I.C.E.T. PIP 0560/98).

\section{REFERENCIAS}

1. Eriksson,O.E. (2006). Outline of Ascomycota. Myconet 12:1-82

2. Currah, R.S. (1985). Taxonomy of the Onygenales: Arthrodermataceae, Gymnoascaceae, Myxotrichaceae and Onygenaceae. Mycotaxon 24:1-216

3. Weitzman, I. \& Summerbell, R. (1995). The Dermatophytes. Clin. Microbiol. Review 8:240-259

4. Rebell, G. \& Taplin, D. (1970). Dermathophytes. Their recognition and identification. $3^{\text {th }}$ ed Coral Gables,Florida, University of Miami Press, USA.

5. Rippon, J,W. (1990). Micología Médica. Hongos y Actinomicetos patógenos. $3^{\text {th }}$ ed. México: Ed. Interamericana, Mc Graw- Hill.

6. Hoog G.S. de.; Guarro, J.; Gené, J. \& Figueras, M.J. (2000). Atlas of Clinical Fungi, $2^{\circ}$ Ed., Centraalboureau voor Schimmelcultures (Ultrecht, The Netherlands)/Universitat Rovira I Virgili (Reus, Spain).

7. Marcano, C. \& Borelli, D. (1983). Tinea unguis caused by Nannizzia gypsea (+). Mycopathologia 81:77-82

8. Romano, C. (1998). Onychomycosis due to Microsporum 
gypseum. Mycoses 41:349-351

9. Sun, P.L. \& Ho, H.T. (2006). Concentric rings: an unusual presentation of tinea corporis caused by Microsporum gypseum. Mycoses 49:150-151

10. Galhardo, M.; Wanke, B.; Reis, R.S.; Oliveira, L.A.; Valle, A. (2004). Disseminated dermatophytosis caused by Microsporum gypseum in an AIDS patient:response to terbinafine and amorolfine. Mycoses 47:238-241

11. Fernandez-Torres, B.; Mayayo, E.; Boronat, J. \& Guarro, J. (2002). Subcutaneous infection by Microsporum gypseum. Br. J. Dermatol. 146:311-313

12. Stockdale P. (1963). The Microsporum gypseum complex (Nannizzia incurvata Stockd., N. gypsea (Nann.) Comb. Nov., N. fulva sp. nov. Sabouraudia 3:114-126

13. Vanbreuseghem, R. (1952). Technique biologique pour l' isolement des dermathophytes du sol. Ann. Soc. Belg. Trop. 32:173-178

14. Weitzman, I.; McGinnis, M.R.; Padhye, A.A. \& Ajello, L. (1986). «The genus Arthroderma and its later synonym Nannizzia. Mycotaxon; XXV:505-518

15. van Gelderen de Komaid, A.A. \& Elías, F. (1979). Presencia de dermatofitos en suelo de escuelas de Tucumán, R. Argentina. Rev. Lat. Am. Microbiol. 20:95-96

16. van Gelderen de Komaid A.A. \& Alvarez, C. (2007). Dermatophytes and other keratinophilic fungi from soil from squares and parks in San Miguel de Tucumán, Argentina. En prensa.
17. Elías, F; van Gelderen de Komaid, A.A.; Pesce de Ruiz Holgado, A. (1978). Micosis superficiales y profundas detectadas en Tucumán entre los años 1965-1975. Rev. Lat. Am. Microbiol. 20:99-105

18. van Gelderen de Komaid, A; Silva, J.O.; Durán, E.; Borges de Kestelman, I.; Runco, R. (1998). Dermatofitias de piel lampiña en Tucumán, VIII Congreso Argentino de Micología, San Miguel de Tucumán, Argentina.

19. Simpanya, M.F. (2000). Dermatophytes: Their taxonomy, ecology and Pathogenicity. In: Kuswaha, R.K.S. \& Guarro, J. (eds.). Biology of Dermatophytes and other keratinophilic fungi. Rev. Iberoam. Micología pp.1-12

20. Hironaga, M.; Tanaka, S. \& Watanabe, S. (1982). Distribution of mating types among clinical isolates of the Microsporum gypseum complex. Mycopathologia 81:77-82

21. Demange, C.; Contet-Audonneau, N.; Kombila, M.; Miegeville, M.; Berthonneau, M.; De Vroey, Ch.; Percebois, G. (1992). Microsporum gypseum complex in man and animals. J. Med. Vet. Mycol. 30:301-308

22. Takashio, M. (1973). Etude de phénomènes de reproduction lié au viellisement et au rajeunissement des cultures de champignons. Ann. Soc. Belge Med. Trop. 53:427-580 